\title{
Frame Based Precoding in Satellite Communications: A Multicast Approach
}

\author{
Dimitrios Christopoulos*, Symeon Chatzinotas* and Björn Ottersten* \\ ${ }^{*}$ SnT - securityandtrust.lu, University of Luxembourg \\ email: \{dimitrios.christopoulos, symeon.chatzinotas, bjorn.ottersten\}@uni.lu
}

\begin{abstract}
In the present work, a multibeam satellite that employs aggressive frequency reuse towards increasing the offered throughput is considered. Focusing on the forward link, the goal is to employ multi-antenna signal processing techniques, namely linear precoding, to manage the inter-beam interferences. In this context, fundamental practical limitations, namely the rigid framing structure of satellite communication standards and the on-board per-antenna power constraints, are herein considered. Therefore, the concept of optimal frame based precoding under per-antenna constraints, is discussed. This consists in precoding the transmit signals without changing the underlying framing structure of the communication standard. In the present work, the connection of the frame based precoding problem with the generic signal processing problem of conveying independent sets of common data to distinct groups of users is established. This model is known as physical layer multicasting to multiple cochannel groups. Building on recent results, the weighted fair per-antenna power constrained multigroup multicast precoders are employed for frame based precoding. The throughput performance of these solutions is compared to multicast aware heuristic precoding methods over a realistic multibeam satellite scenario. Consequently, the gains of the proposed approach are quantified via extensive numerical results.
\end{abstract}

Index Terms-Frame based Precoding; Physical layer Multigroup Multicasting; Per-antenna Power Constraints; Multicast Aware MMSE; Weighted Max Min Fair Optimization;

\section{INTRODUCTION \& RELATED WORK}

The spatial degrees of freedom offered by the multibeam satellite antenna are a valuable resource towards efficiently reusing the user link bandwidth. Advanced transmit signal processing techniques, namely beamforming (or equivalently precoding), are currently employed to optimize the performance of the multi-antenna transmitters without compromising the complexity of single antenna receivers. This allows for more aggressive frequency reuse schemes, towards the efficient utilization of the available spectrum and thus the increase of the overall system throughput. As always, however, the benefits of these advanced schemes come with a cost. The most inhibiting requisite for the application of linear precoding is the knowledge of the vector channel state information (CSI) at the transmitter. Assuming readily available CSI at the transmit side, full-frequency re-use schemes are foreseen to boost the throughput of the next generation broadband interactive high throughput multibeam satellite systems, by the means of linear precoding [1].

Conventionally, the channel capacity achieving precoding design assumes independent symbols, each addressed to a different single user. This allows for a channel by channel calcu- lation of the precoding matrices. However, such an assumption cannot apply in a satellite scenario. The framing structure of optimized with the inherent attributes of the satellite channel of legacy communication standards, imposes specific constraints in the practical implementation of precoding. The physical layer design of DVB - S2 [2] and DVB - S2X [3], encompasses long forward error correction codes (FEC) to cope with noise limited channels and long propagation delays. Therefore, as the framing of multiple users per transmission is emanated to guarantee scheduling efficiency, the particularly long FEC frames inhibit precoding. Consequently, the traditional assumption of a single user terminal (UT) per transmission is alleviated and the application of precoding methods in future satellite systems relies on frame-by-frame precoding [1].

An toy example of the application of linear precoding in satcoms is given in Fig. 1. Therein four frames belonging to two subsequent transmissions are shown. Each transmitted frame addresses multiple users. A symbol denoted as $s_{i}$ is addressed to the $i$-th user of the corresponding frame. One should bear in mind that bit and symbol interleaving take place in a frame, while user data payloads are not always equal, as depicted in the second transmission in Fig. 1. In frames 3 and 4, different amount of data is transmitted to each user. It should be stressed that symbols denoted as $s_{a}$ are not carrying identical data. To simplify the analysis, in the following it will be assumed that in each beam, an equal number of UTs is co-scheduled in each frame. Hence, the first transmission instance of Fig. 1 will be modeled. Such an assumption can be realized in a practical system via the use of dummy data to fill frames. Also, the frames are of constant size and transmissions amongst the beams are perfectly synchronized. These assumptions are in line with the latest evolution of DVB-S2X [3] [4].

The purpose of the present work is to establish the connection of frame based precoding with the generic, physical layer multicasting problem [5]. More specifically, physical layer (PHY) multicasting to multiple co-channel groups [6] can provide the theoretically optimal precoders when a multiantenna transmitter conveys independent sets of common data to distinct groups of users. This scenario is known as multigroup multicasting. The connection of the frame based precoding problem with the PHY multigroup multicasting is clear under the following considerations. Multicasting is based on the assumption that the same information is transmitted to multiple receivers. This leads to designing a precoding vector matched to more than one channel vectors. From a different 


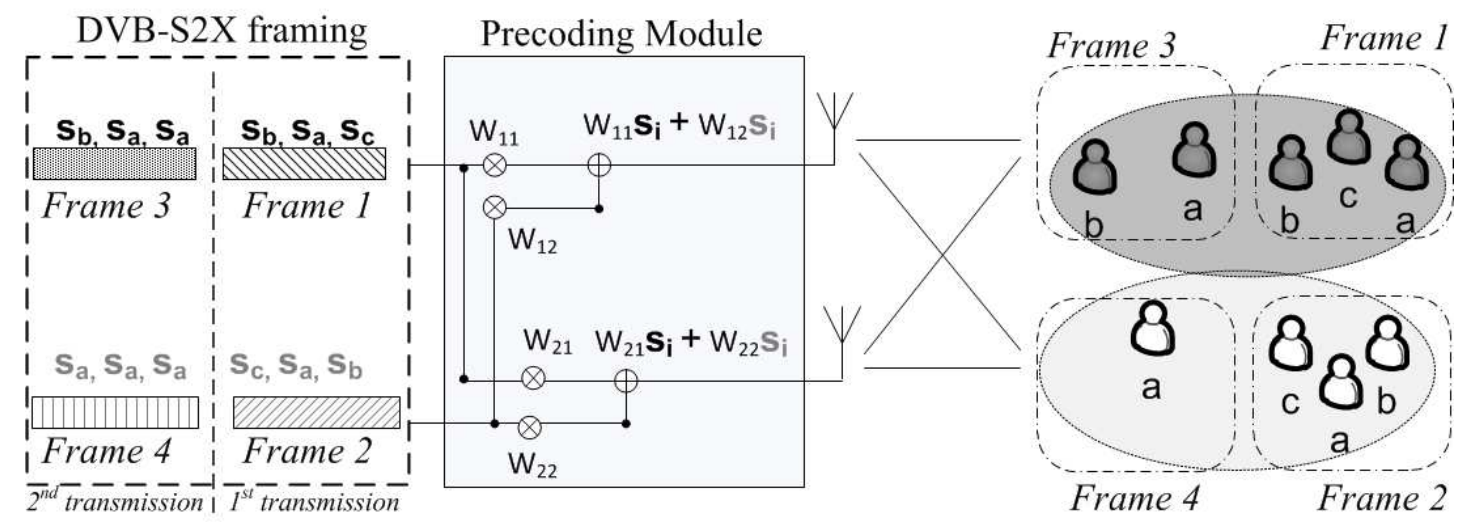

Fig. 1. Frame based precoding transmitter: a toy example.

perspective, the framing structure of communication standards imposes that the same precoder will apply over the symbols of more than one user belonging in the same frame. Since each frame is received and decoded by all co-group users, the design of an optimal frame based precoder is given by solving a multicast multigroup optimization problem. Thus, multicasting allows for an analytically formal modeling of the problem. Therefore, in the context of frame based precoding, the fact that the same precoder needs to apply to the different data of many receivers due to the framing constraint, leads to a multicast consideration.

The second practical constraint tackled herein involves the on-board per-antenna constraints imposed by conventional multibeam communication payloads. The lack of flexibility in sharing energy resources amongst the antennas of the transmitter is in general a common scenario in multi-antenna systems where individual amplifiers per transmit antenna, are employed. Despite the fact that flexible amplifiers could be incorporated in some cases, specific communication systems cannot afford this design. Typical per-antenna power limited systems can be found in multibeam satellite communications [1], where flexible on board payloads are difficult to implement. Other examples can be found in distributed antenna systems, such as cooperative satellite constellations or swarms of cooperative nano-satellites.

The optimal transmit beamforming in the minimum total transmit power sense, assuming channel based precoding, was initially derived under sum power constraints (SPC) over all the transmit antennas in [7], [8]. Per-antenna transmit power constraints (PACs) were considered later on, in [9]. Moreover, the multiuser downlink beamforming problem in terms of maximizing the minimum SNIR, was optimally solved in [10]. The goal of the later formulation is to increase the fairness of the system by boosting the SNIR of the user that is further away from a targeted performance. Hence, the problem is commonly referred to as max-min fair. Amid the extensive literature on multigroup multicast beamforming [6], the derivation of the optimal multigroup multicast precoders when a maximum limit is imposed on the transmitted power of each antenna is non trivial. In this direction, the weighted max-min fair multigroup multicast beamforming for a perantenna power constrained system has been derived in [11],
[12]. In the present work, this solution is applied in the context of multibeam satellite systems. This allows for the frame based precoding problem for satellite communications to be formulated in an analytically tractable manner.

The rest of the paper is structured as follows. The description of the multigroup multicast satellite system model is given in Sec. II. Heuristic approaches to solve this issue are discussed in Sec. III-A. The multicast multigroup optimization problem definition is described in Sec. III-B In Sec. IV, the performance of the proposed technique over the multibeam satellite system is evaluated. Finally, Sec. $\mathrm{V}$ concludes the paper and paves the way forward.

Notation: In the remainder of this paper, bold face lower case and upper case characters denote column vectors and matrices, respectively. The operators $(\cdot)^{\mathrm{T}},(\cdot)^{\dagger},|\cdot|$ and $\|\cdot\|_{2}$, correspond to the transpose, the conjugate transpose, the absolute value and the Frobenius norm operations over matrices and vectors, while $[\cdot]_{i j}$ denotes the $i, j$-th element of a matrix.

\section{SySTEM MODEL}

Let us focus on a multi-user (MU) multiple input single output (MISO) multicast system. Assuming a single transmitter, let $N_{t}$ denote the number of transmitting elements and $N_{u}$ the total number of users served. The input-output analytical expression will read as $y_{i}=\mathbf{h}_{i}^{\dagger} \mathbf{x}+n_{i}$, where $\mathbf{h}_{i}^{\dagger}$ is a $1 \times N_{t}$ vector composed of the channel coefficients (i.e. channel gains and phases) between the $i$-th user and the $N_{t}$ antennas of the transmitter, $\mathrm{x}$ is the $N_{t} \times 1$ vector of the transmitted symbols and $n_{i}$ is the independent complex circular symmetric (c.c.s.) independent identically distributed (i.i.d) zero mean Additive White Gaussian Noise (AWGN) measured at the $i$-th user's receive antenna.

By defining a single multicast group per beam, in each transmission, the multigroup multicast model of [6] is realized. Thus, a total of $G=N_{t}$ multicast groups are assumed, with $\mathcal{I}=\left\{\mathcal{G}_{1}, \mathcal{G}_{2}, \ldots \mathcal{G}_{G}\right\}$ the collection of index sets and $\mathcal{G}_{k}$ the set of users that belong to the $k$-th multicast group, $k \in\{1 \ldots G\}$. Each user belongs to only one group, thus $\mathcal{G}_{i} \cap \mathcal{G}_{j}=\varnothing, \forall i, j \in\{1 \cdots G\}$. Let $\mathbf{w}_{k} \in \mathbb{C}^{N_{t} \times 1}$ denote the precoding weight vector applied to the transmit antennas to beamform towards the $k$-th group. Let us also denote the number of users per group as $\rho=N_{u} / G$. By collecting all 
user channels in one channel matrix, the general linear signal model in vector form reads as

$$
\mathbf{y}=\mathbf{H x}+\mathbf{n}=\mathbf{H W} \mathbf{s}+\mathbf{n}
$$

where $\mathbf{y}$ and $\mathbf{n} \in \mathbb{C}^{N_{u}}, \mathbf{x} \in \mathbb{C}^{N_{t}}$ and $\mathbf{H} \in \mathbb{C}^{N_{u} \times N_{t}}$. The multigroup multicast scenario imposes a precoding matrix $\mathbf{W} \in \mathbb{C}^{N_{t} \times N_{t}}$ that includes as many precoding vectors (i.e columns) as the number of groups $\left(G=N_{t}\right)$. This is the number of independent symbols transmitted, i.e. $\mathbf{s} \in \mathbb{C}^{N_{t}}$. The assumption of independent information transmitted to different groups implies that the symbol streams $\left\{s_{k}\right\}_{k=1}^{G}$ are mutually uncorrelated and the total power radiated from the antenna array is equal to

$$
P_{\text {tot }}=\sum_{k=1}^{N_{t}} \mathbf{w}_{k}^{\dagger} \mathbf{w}_{k}=\operatorname{Tr}\left(\mathbf{W} \mathbf{W}^{\dagger}\right)
$$

where $\mathbf{W}=\left[\mathbf{w}_{1}, \mathbf{w}_{2}, \ldots \mathbf{w}_{G}\right]$. The power radiated by each antenna element is a linear combination of all precoders and reads as [9]

$$
P_{n}=\left[\sum_{k=1}^{N_{t}} \mathbf{w}_{k} \mathbf{w}_{k}^{\dagger}\right]_{n n}=\left[\mathbf{W} \mathbf{W}^{\dagger}\right]_{n n},
$$

where $n \in\left\{1 \ldots N_{t}\right\}$ is the antenna index. The fundamental difference between the SPC of [6] and the proposed PAC is clear in (3), where instead of one, $N_{t}$ constraints are realized, each one involving all the precoding vectors.

\section{A. Equivalent Channel Model}

Assuming an equal number of groups and antennas results to a square precoding matrix, as seen in the previous section. Therefore, an alternative, simplified channel model in the fashion of [1] and [?] can also be adopted towards providing a more tractable representation. To facilitate the comprehension of system model, let us define multiple square channel matrices $\mathbf{H}_{[i]}$. The index $[i]$ corresponds to the different UTs per beam that need to be served by the same frame, i.e. $i=1, \ldots \rho$. Each matrix corresponds to a "a single user-perbeam" instance, which is the common assumption in satellite precoding literature (e.g. [13] and the references therein). To model the frame based precoding constraint, the general inputoutput signal model can be defined as [1]:

$$
\mathbf{y}_{[i]}=\mathbf{H}_{[i]} \mathbf{x}_{[i]}+\mathbf{n}_{[i]}=\mathbf{H}_{[i]} \mathbf{W} \mathbf{s}_{[i]}+\mathbf{n}_{[i]}
$$

where $\mathbf{y}, \mathbf{x}, \mathbf{n}, \mathbf{s} \in \mathcal{C}^{N_{t}}$, with $\mathcal{E}\|\mathbf{n}\|^{2}=\sigma^{2}$ and $\mathcal{E}\|\mathbf{s}\|^{2}=1$, while $\mathbf{H}_{[i]} \in \mathcal{C}^{N_{t} \times N_{t}}$ is a one-user-per-beam instance of the total non-square channel matrix. Also, since an equal number of antennas and groups is assumed, $N_{u}=\rho \cdot N_{t}$. The above definition allows for the calculation of one equivalent precoder $\mathbf{W}=f\left(\mathbf{H}_{[i]}\right)$. The function $f$ can be chosen according to the design criteria [?].

\section{B. Multibeam Satellite Channel}

The above general system model, is herein applied over a multibeam satellite channel explicitly defined as follows. A 245 beam pattern that covers Europe is employed [14]. The

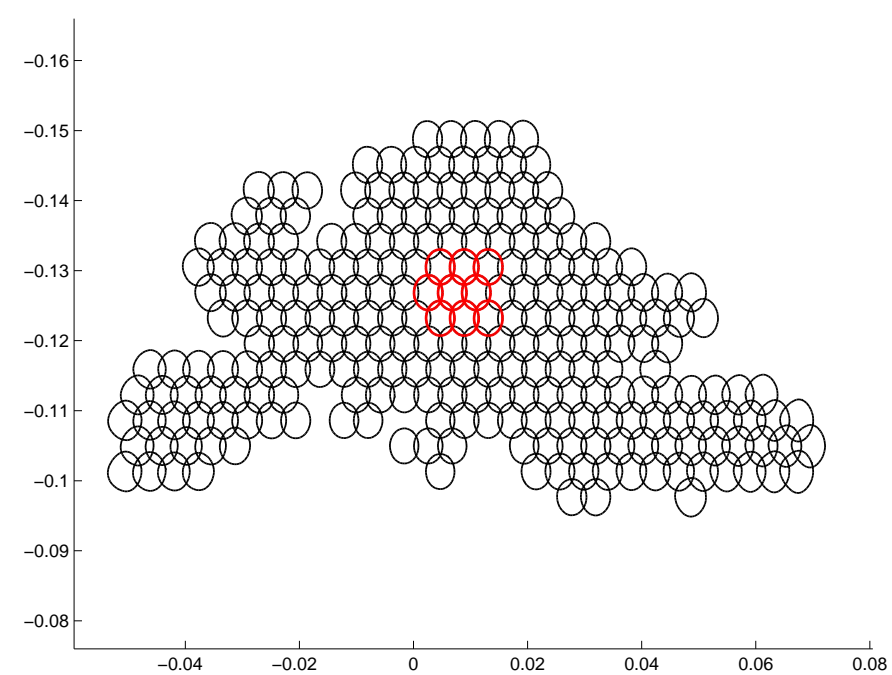

Fig. 2. Plot of the coverage area with the 9 selected beams

multibeam radiation pattern is depicted in Fig. 2 A complex channel matrix that models the link budget of each UT as well as the phase rotations induced by the signal propagation and the payload is employed. In more detail, starting from the model followed in [15], the total channel matrix $\mathbf{H} \in \mathbb{C}^{N_{u} \times N_{t}}$ is generated as

$$
\mathbf{H}=\mathbf{\Phi}_{p} \mathbf{B},
$$

and models the multibeam antenna pattern as well as the signal phase due to different propagation paths between the users. The real matrix $\mathbf{B} \in \mathbb{R}^{N_{u} \times N_{t}}$ models the satellite antenna radiation pattern, the path loss, the receive antenna gain and the noise power. Its $i, j$-th entry is given by :

$$
b_{i j}=\left(\frac{\sqrt{G_{R} G_{i j}}}{4 \pi\left(d_{k} \cdot \lambda^{-1}\right) \sqrt{\kappa T_{c s} W}}\right),
$$

with $d_{k}$ the distance between the $k$-th UT and the satellite (slant-range), $\lambda$ the wavelength, $\kappa$ the Boltzman constant, $T_{c s}$ the clear sky noise temperature of the receiver, $W$ the user link bandwidth, $G_{R}$ the receiver antenna gain and $G_{i j}$ the multibeam antenna gain between the $i$-th single antenna UT and the $j$-th on board antenna ( $=$ feed). Hence, the beam gain for each antenna-UT pair, depends on the antenna pattern and on the user position. A fundamental assumption in multibeam satellite channels lies in assuming that one user will have the same phase between all transmit antennas due to the long propagation path [13], [15]-[17]. The identical phase assumption between one UT and all transmit feeds is supported by the relatively small distances between the transmit antennas and the long propagation distance of all signals to a specific receiver. However, this assumption discards any phase introduced by the on-board equipment due to imperfections and/or different on board propagation path: 1 . Hence, in (5) the diagonal square matrix $\boldsymbol{\Phi}_{p} \in \mathbb{C}^{N_{u} \times N_{u}}$ is generated as $\left[\boldsymbol{\Phi}_{p}\right]_{k k}=e^{j \phi_{k}}, \forall k=1 \ldots N_{u}$ where $\phi_{k}$ is a uniform random variable in $(0,2 \pi]$ and $\left[\boldsymbol{\Phi}_{p}\right]_{k n}=0, \forall k \neq n$.

\footnotetext{
${ }^{1}$ More elaborate signal phase assumptions that consider the on-board payload imperfections, as well as phase offsets introduced by the receiver equipment, will be considered in future extensions of this work.
} 
TABLE I

Link Budget \& Simulation PARAMETERS

\begin{tabular}{l|l} 
Parameter & Value \\
\hline Frequency Band & $\mathrm{K}(20 \mathrm{GHz})$ \\
UT clear sky temp, $T_{c s}$ & $235.3 \mathrm{~K}$ \\
User Link Bandwidth, $B_{u}$ & $500 \mathrm{MHz}$ \\
Output Back Off, OBO & $5 \mathrm{~dB}$ \\
On board Power, $P_{t o t}$ & $55 \mathrm{dBW}$ \\
Roll off, $\alpha$ & 0.20 \\
UT antenna Gain, $G_{R}$ & $40.7 \mathrm{dBi}$ \\
Multibeam Antenna Gain, $G_{i j}$ & Ref: [14] \\
\hline
\end{tabular}

\section{Multigroup Multicast Precoding}

\section{A. Multicast Aware MMSE: Average Precoding}

The optimal linear precoder $\mathbf{W}=f\left(\mathbf{H}_{[i]}\right), i=1 \ldots \rho$ in the minimum mean square error sense, with more users than transmit antennas is considered in this section. Under the constraint of designing a linear MMSE precoder $\mathbf{W} \in \mathbb{C}^{N_{t} \times N_{t}}$ for multiple channels, i.e. $\mathbf{H} \in \mathbb{C}^{N_{u} \times N_{t}}$ with $N_{u}>N_{t}$ the solution is not straightforward. Following the equivalent channel notation of Sec. II-A the problem of minimizing the MSE between the transmitted and the received signals over a noisy channel is formalized as

$$
\begin{aligned}
& \mathbf{W}=\arg \min \mathcal{E}\left\|\left[\begin{array}{c}
\mathbf{H}_{[1]} \\
\mathbf{H}_{[2]} \\
\vdots \\
\mathbf{H}_{[\rho]}
\end{array}\right][\mathbf{W}][\mathbf{s}]+\left[\begin{array}{c}
\mathbf{n}_{[1]} \\
\mathbf{n}_{[2]} \\
\vdots \\
\mathbf{n}_{[\rho]}
\end{array}\right]-\left[\begin{array}{c}
\mathbf{s} \\
\mathbf{s} \\
\vdots \\
\mathbf{s}
\end{array}\right]\right\|^{2} \\
& \text { s.t. } \mathcal{E}\|\mathbf{W} \mathbf{s}\|^{2}=P_{n},
\end{aligned}
$$

for the case that we need to serve $\rho=N_{u} / G$ users in each group using the same precoder. Problem (7) can be analytically solved, in the fashion of [18], by noting that the cost function is the following sum:

$$
\begin{aligned}
C_{\text {] }}= & \operatorname{Tr}\left[\left(\mathbf{H}_{[1]} \mathbf{W}-\mathbf{I}\right)\left(\mathbf{H}_{[1]} \mathbf{W}-\mathbf{I}\right)^{\dagger}\right]+\beta \operatorname{Tr}\left[\mathbf{W} \mathbf{W}^{\dagger}\right] \\
& +\ldots \\
& \operatorname{Tr}\left[\left(\mathbf{H}_{[\rho]} \mathbf{W}-\mathbf{I}\right)\left(\mathbf{H}_{[\rho]} \mathbf{W}-\mathbf{I}\right)^{\dagger}\right]+\beta \operatorname{Tr}\left[\mathbf{W} \mathbf{W}^{\dagger}\right] \\
= & \sum_{i=1}^{\rho} \operatorname{Tr}\left[\left(\mathbf{H}_{[i]} \mathbf{W}-\mathbf{I}\right)\left(\mathbf{H}_{[i]} \mathbf{W}-\mathbf{I}\right)^{\dagger}\right] \\
& +\rho \beta \operatorname{Tr}\left[\mathbf{W} \mathbf{W}^{\dagger}\right]
\end{aligned}
$$

where $\beta=\sigma^{2} / P_{n}$. By differentiation we get

$$
\begin{aligned}
& \nabla_{\mathbf{W}} C(\mathbf{W})=0 \Leftrightarrow \\
& \mathbf{W}\left(\sum_{i=1}^{\rho} \mathbf{H}_{[i]}^{\dagger} \mathbf{H}_{[i]}+\rho \beta \mathbf{I}\right)=\sum_{i=1}^{\rho} \mathbf{H}_{[i]}^{\dagger},
\end{aligned}
$$

Thus the general solution reads as

$$
\mathbf{W}=\left(\frac{1}{\rho} \sum_{i=1}^{\rho} \mathbf{H}_{[i]}^{\dagger} \mathbf{H}_{[i]}+\beta \mathbf{I}\right)^{-1} \frac{1}{\rho} \sum_{i=1}^{\rho} \mathbf{H}_{[i]}^{\dagger}
$$

Following a different derivation methodology, this result was firstly reported in [19].

Remark 1: Under the assumption of Rayleigh fading, the elements of $\mathbf{H}$ are independent zero mean complex Gaussian instances. Subsequently, due to the central limit theorem, as the number of users per group $\rho$ increases then the precoder will tend to zero:

$$
\lim _{\rho \rightarrow \infty} \frac{1}{\rho} \sum_{i=1}^{\rho} \mathbf{H}_{[i]}=0 .
$$

The implications of Remark 1 can be seen when the system dimensions grow large and the channel matrices tend to be modeled as zero mean random variables. The main result is that the system performance will degrade as the number of users per group increases. Assuming a fixed number of groups, the degradation as the number of users increases has only been examined hitherto via simulations [6], [19]. Herein, an analytical proof for this result has been provided. Moreover, remembering that $\rho=N_{u} / G$, for a fixed number of users the performance is expected to degrade as the number of groups increases. Since each user belongs to only one group, the maximum number of groups is bounded by $N_{u}$. Hence, the best performance is expected for a one user per group configuration. In other words, multicasting is expected to perform worst, in terms of precoding gain, over unicasting. An expected result, if one considers that in multicasting the degrees of freedom at the transmit side are reduced.

The above results provide a multicast aware MMSE solution for the calculation of the precoding matrix. However, the main drawback of this solution is that it does not account for the practical per-antenna constraints. The simplest heuristic to overcome this obstacle is to re-scale the solution so that the per-antenna constraints are not violated [?]. Despite the fact that such an operation invalidates the MMSE optimality of the solution, it provides a low complexity heuristic method to design the precoder. Re-scaling is achieved by multiplying each line of the precoding matrix with the square root of the inverse level of power over satisfaction of the corresponding antenna.

\section{B. Multicast Multigroup Beamforming under PACs}

The MMSE solution described in the previous section is one candidate linear precoding method. Another approach, namely the weighted max-min fair optimization of [11], [12] is considered hereafter. The main benefit of this approach lies in the optimality of the solution as far as the PACs are concerned. The weighted max-min fair problem with PACs reads as

$$
\begin{aligned}
& \mathcal{F}: \max _{t,\left\{\mathbf{w}_{k}\right\}_{k=1}^{G}} t \\
& \text { subject to } \frac{1}{\gamma_{i}} \frac{\left|\mathbf{w}_{k}^{\dagger} \mathbf{h}_{i}\right|^{2}}{\sum_{l \neq k}^{G}\left|\mathbf{w}_{l}^{\dagger} \mathbf{h}_{i}\right|^{2}+\sigma_{i}^{2}} \geq t, \\
& \forall i \in \mathcal{G}_{k}, k, l \in\{1 \ldots G\}, \\
& \text { and to } \quad {\left[\sum_{k=1}^{G} \mathbf{w}_{k} \mathbf{w}_{k}^{\dagger}\right]_{n n} \leq P_{n}, } \\
& \forall n \in\left\{1 \ldots N_{t}\right\},
\end{aligned}
$$

where $\mathbf{w}_{k} \in \mathbb{C}^{N_{t}}$ and $t \in \mathbb{R}^{+}$. Problem $\mathcal{F}$ receives as inputs the PAC vector $\mathbf{p}=\left[P_{1}, P_{2} \ldots P_{N_{t}}\right]$ and the target SNIRs vector $\mathbf{g}=\left[\gamma_{1}, \gamma_{2}, \ldots \gamma_{N_{u}}\right]$. Following the common 
in the literature notation for ease of reference, the optimal objective value of $\mathcal{F}$ will be denoted as $t^{*}=\mathcal{F}(\mathbf{g}, \mathbf{p})$ and the associated optimal point as $\left\{\mathbf{w}_{k}^{\mathcal{F}}\right\}_{k=1}^{G}$. A common SNIR target between multiple users is a special case of the above general formulation. Of particular interest is the case where the users that belong in the same group share the same target i.e. $\gamma_{i}=\gamma_{k}, \forall i \in \mathcal{G}_{k}, k \in\{1 \ldots G\}$, since the performance of all co-group users will be defined by the worst user in the group. Towards solving this elaborate problem via the means of convex optimization, the principles of Semi-definite Relaxation (SDR) and Gaussian randomization [20] need to be combined with bisection. The detailed solution of this problem is given in [11], [12], and is omitted herein for shortness. Detailed discussions on the complexity of this method are also given therein. For the purposes of this work, it is only mentioned that the complexity is higher than the multicast aware heuristics, but it still remains polynomial in order. The exact determination of the trade-off between complexity increase and performance gains is left for future work. The optimally fair solutions are compared to the MMSE based heuristics in the following.

\section{Performance Evaluation \& Application}

Extensive numerical results that exhibit the applicability of precoding in satellite communications are presented. To the end of providing accurate results, the simulation setup of [1] is employed. The simulation parameters are summarized in Tab. [1 The achievable spectral efficiency of the $k$-th user is directly linked with its $\mathrm{SNIR}_{k}$ through the DVB - S2 [2] achievable spectral efficiency 2. More importantly, to account for adaptive coding and modulation (ACM) and the fact that a single modulation and coding scheme (MODCOD) is applied to each frame, the $\rho$ UTs that are simultaneously served by the same frame are assumed to be using the MODCOD corresponding to lowest SNIR value in the group. This consideration is inline with the common multicast consideration that the user with the lowest rate in each group will determine the performance of the group. The multibeam satellite antenna pattern has been provided in [1], [14]. From the 245 beams used to cover Europe, the focus herein is on a cluster of 9 beams, as depicted in Fig. 2. This assumption is inline with future multi-gateway considerations, where precoding will be performed in each gateway separately [22]. Perfect channel state information is assumed throughout this work. The complex channel coefficients are generated as described in Sec. [I-B, where only the phases due to different propagation paths between the satellite and users are assumed [15]. Herein, the interferences from adjacent clusters are not accounted for, since the purpose is to give a relative comparison between the possible precoding methods rather than an absolute evaluation of the total system throughput. For ease of reference, however, the results are given on a per beam basis. Finally, it needs to be stressed, that since the purpose of this work is to establish the most promising precoding method, no user scheduling is considered. Therefore, the results presented hereafter are averaged over

\footnotetext{
${ }^{2}$ More up-to-date DVB - S2X spectral efficiency mapping has been considered in [21].
}

uniformly random over the coverage distributed users, when a random schedule is considered in each frame.

The per beam achievable throughput with respect to an increasing on board available power budget for the conventional 4 color frequency reuse scheme and the two proposed precoding methods is given in Fig. 3. For a nominal on board power of $55 \mathrm{dBW}$, the weighted fair solution achieves $42 \%$ improvement over the conventional system, while the heuristic average precoder $21 \%$. In the same figure, the substantial gain of the proposed techniques with respect to an increasing power budget is also presented. This is gain identical for both precoding methods. Fig. 4 presents the per beam throughput when four users per frame are considered. For this setting, the heuristic sub-optimal system performs worst than the conventional systems. However, the multicast approach still manages to achieve some gains $(6 \%)$.

To investigate the sensitivity of all methods to the frame dimensions, the per beam throughput is plotted with respect to an increasing number of users per frame in Fig. 5. The performance degradation of all precoding methods with the increasing number of users per frame is apparent. This expected result [11] is justified by Remark 1. This is intuitively expected by the inherent constraints of linear precoding methods. As the number of users increases, the transmit spatial degrees of freedom do not suffice to manage interferences and the performance is degraded. Nevertheless, the optimal multicast scheme manages to maintain gains over the conventional systems for up to five users per frame, whereas the heuristic scheme provides gains for up to two users per frame.

In Figs. 6 and 7 the per user rate distribution over the coverage area for two and four users per frame respectively is plotted. In these figures, insights on the origins of the gains of the optimal multicast approach are gained. The fairness optimization, reduces the variability of the SNIR across the coverage area and consequently inside each frame. This results in better utilization of resources since similar in terms of SNIRs users are served by the same frame. On the contrary, the MMSE precoding approach exhibits high SNIR variability. Hence, users with different SNIRs are scheduled in the same frame and their performance is compromised by the performance of the worst user. Additionally, many users are driven to the unavailability region, since their SNIR is lower than the minimum value that the available MODCODs can support. As depicted in Figs. 6 and 7 with heuristic MMSE precoding, more than $15 \%$ and $30 \%$ of users experience unavailability incidents over the coverage area respectively and therefore receive zero rate.

\section{Conclusions \& Future Work}

In the present work, the optimal in a fairness sense, perantenna power constrained, multigroup multicast linear precoding vectors have been applied in a multibeam satellite transmitter configured to operate in full-frequency reuse. The throughput performance of the weighted fair multigroup multicast precoding is compared to the heuristic multicast aware MMSE solutions re-scaled to respect the PACs. Simulation results over an accurate multibeam satellite scenario exhibit the 


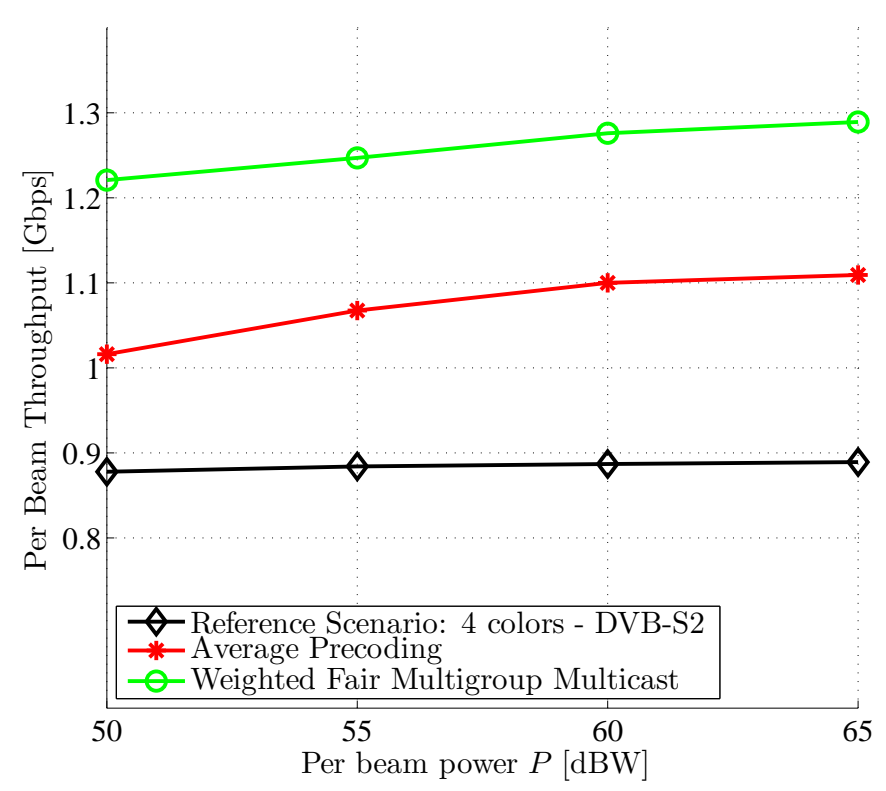

Fig. 3. Per beam throughput performance versus increasing on board power for $\rho=2$ users per frame.

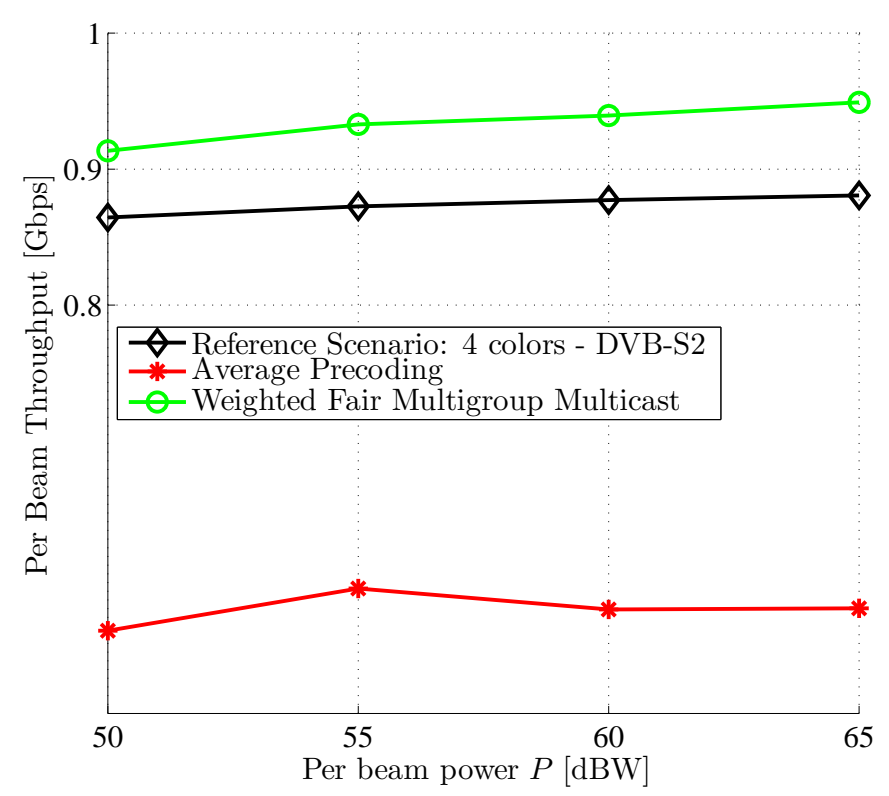

Fig. 4. Per beam throughput performance versus increasing on board power for $\rho=4$ users per frame.

superiority of the multigroup multicast solution over heuristic precoding methods. Insights on the origin of this result are provided. Finally, a sensitivity analysis with respect to system design parameters reveals the limits of the herein considered precoding methods.

Extensions of this work [21], [23] include a sum-rate maximizing precoding design, adapted to the needs of satellite communications. Also, the multiuser gains offered by proper user scheduling are gleaned in [21] towards establishing the applicability of precoding in satellite communications.

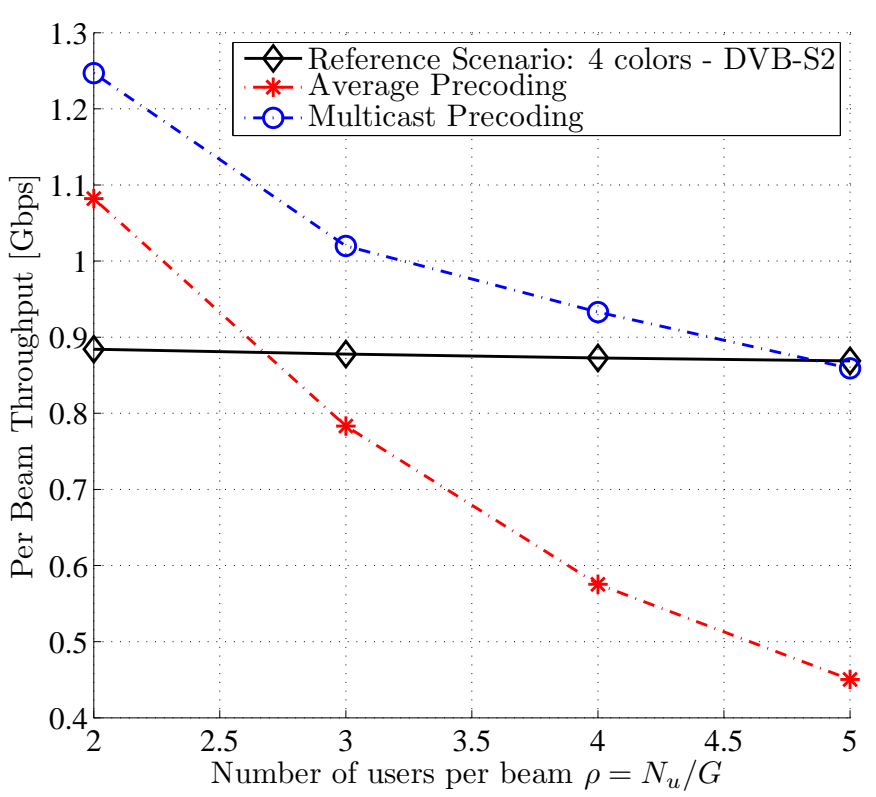

Fig. 5. Per beam throughput versus number of users per frame, for $P=$ 55 dBW.

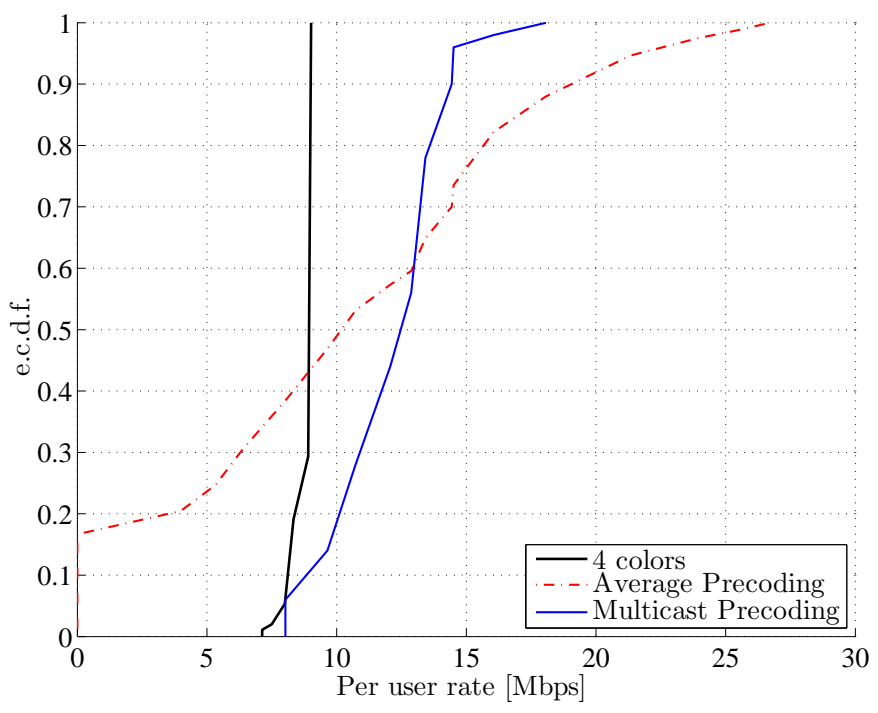

Fig. 6. Per user rate distribution over the coverage for $P=55 \mathrm{dBW}$ and $\rho=2$ users per frame.

\section{ACKNOWLEDGEMENT}

This work was partially supported by the National Research Fund, Luxembourg under the CORE projects " $\mathrm{CO}^{2} \mathrm{SAT}$ : Cooperative and Cognitive Architectures for Satellite Networks" and "SEMIGOD: SpEctrum Management and Interference mitiGation in cOgnitive raDio satellite networks". The authors would like to thank the work of the anonymous reviewers that greatly improved the presentation of this work. Acknowledgements are due to the European Space Agency and specifically Dr. P.-D. Arapoglou and Dr. A. Ginesi for the definition of the frame based precoding problem in satellite communications. 


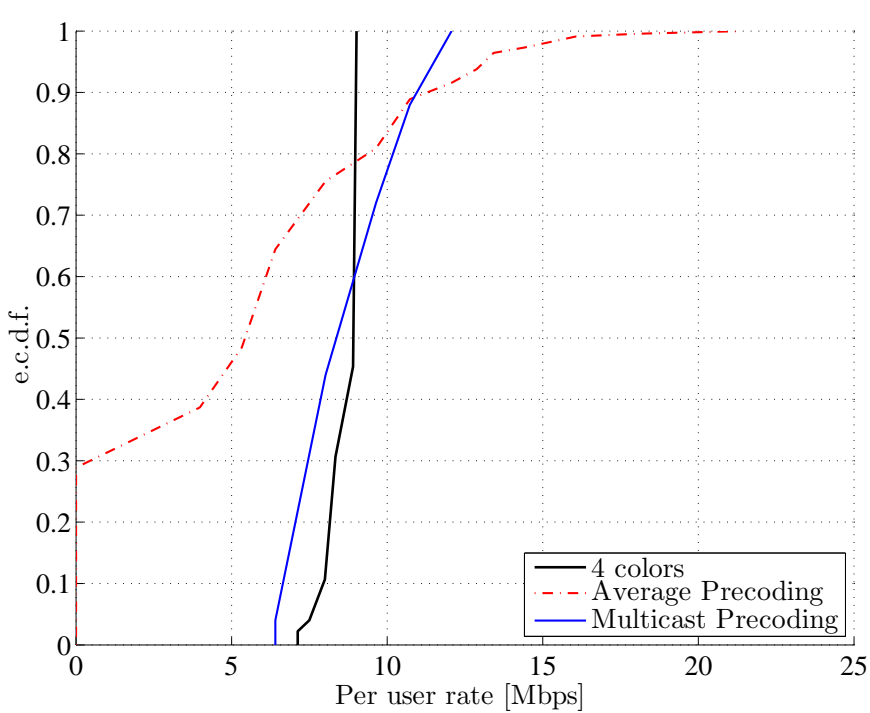

Fig. 7. Per user rate distribution over the coverage for $P=55 \mathrm{dBW}$ and $\rho=4$ users per frame.

\section{REFERENCES}

[1] D. Christopoulos, P.-D. Arapoglou, S. Chatzinotas, and B. Ottersten, "Linear precoding in multibeam satcoms: Practical constraints," in 31st AIAA International Communications Satellite Systems Conference (ICSSC), Florence, IT, Oct. 2013.

[2] ETSI EN 302307 V1.1.2, "Digital video broadcasting (DVB); second generation framing structure, channel coding and modulation systems for broadcasting, interactive services, news gathering and other broad-band satellite applications (DVB-S2), european broadcasting union (EBU)."

[3] DVB Blue Book A83-2, "Second generation framing structure, channel coding and modulation systems for broadcasting, interactive services, news gathering and other broadband satellite applications; part II: S2extensions (S2X)."

[4] A. Morello, "DVB-Sx: The evolution of the (satellite systems) species," in Proc. of 31st AIAA International Communications Satellite Systems Conference (ICSSC), Florence, IT, Oct. 2013.

[5] N. Sidiropoulos, T. Davidson, and Z.-Q. Luo, "Transmit beamforming for physical-layer multicasting," IEEE Trans. Signal Process., vol. 54, no. 6, pp. 2239-2251, 2006.

[6] E. Karipidis, N. Sidiropoulos, and Z.-Q. Luo, "Quality of service and max-min fair transmit beamforming to multiple cochannel multicast groups," IEEE Trans. Signal Process., vol. 56, no. 3, pp. 1268-1279, 2008.

[7] M. Bengtsson and B. Ottersten, "Optimal and suboptimal transmit beamforming," in Handbook of Antennas in Wireless Communications. CRC Press, 2001, pp. 18-1-18-33, qC 20111107.
[8] — , "Optimal downlink beamforming using semidefinite optimization," in Proc. of Annual Allert. Conf. on Commun. Control and Computing, vol. 37. Citeseer, 1999, pp. 987-996.

[9] W. Yu and T. Lan, "Transmitter optimization for the multi-antenna downlink with per-antenna power constraints," IEEE Trans. Signal Process., vol. 55, no. 6, pp. 2646-2660, June 2007.

[10] M. Schubert and H. Boche, "Solution of the multiuser downlink beamforming with individual SINR constraints," IEEE Trans. Veh. Technol., vol. 53, no. 1, pp. 18-28, 2004.

[11] D. Christopoulos, S. Chatzinotas, and B. Ottersten, "Multicast multigroup beamforming under per-antenna power constraints," in Proc. of IEEE International Communications Conference (ICC), Sydney, AU, Jul. 2014.

[12] — - "Weighted fair multicast multigroup beamforming under perantenna power constraints," IEEE Trans. Signal Process., 2014, under review.

[13] D. Christopoulos, S. Chatzinotas, G. Zheng, J. Grotz, and B. Ottersten, "Linear and non-linear techniques for multibeam joint processing in satellite communications," EURASIP J. on Wirel. Commun. and Networking 2012, 2012:162. [Online]. Available: http://jwcn.eurasipjournals.com/content/2012/1/162

[14] Satellite Network of Experts (SatNEx) 3, "Call of order 2-task 1: Fair comparison and combination of advanced interference mitigation techniques," ESA Contract 23089/10/NL/CPL.

[15] G. Zheng, S. Chatzinotas, and B. Ottersten, "Generic optimization of linear precoding in multibeam satellite systems," IEEE Trans. Wireless Commun., vol. 11, no. 6, pp. $2308-2320$, Jun. 2012.

[16] D. Christopoulos, S. Chatzinotas, M. Matthaiou, and B. Ottersten, "Capacity analysis of multibeam joint decoding over composite satellite channels," in Proc. of 45th Asilomar Conf. on Signals, Systems and Computers, Pacific Grove, CA, Nov. 2011, pp. $1795-1799$.

[17] D. Christopoulos, J. Arnau, S. Chatzinotas, C. Mosquera, and B. Ottersten, "Mmse performance analysis of generalized multibeam satellite channels," IEEE Commun. Lett., vol. 17, no. 7, pp. 1332-1335, 2013.

[18] R. L.-U. Choi and R. Murch, "New transmit schemes and simplified receivers for MIMO wireless communication systems," IEEE Trans. Wireless Commun., vol. 2, no. 6, pp. 1217-1230, 2003.

[19] Y. C. B. Silva and A. Klein, "Linear transmit beamforming techniques for the multigroup multicast scenario," IEEE Trans. Veh. Technol., vol. 58, no. 8, pp. 4353-4367, 2009.

[20] Z.-Q. Luo, W.-K. Ma, A.-C. So, Y. Ye, and S. Zhang, "Semidefinite relaxation of quadratic optimization problems," IEEE Signal Processing Mag., vol. 27, no. 3, pp. 20-34, 2010.

[21] D. Christopoulos, S. Chatzinotas, and B. Ottersten, "Full frequency reuse multibeam satellite systems: Frame based precoding and user scheduling," IEEE Trans. Wireless Commun., 2014, under preparation.

[22] S. Chatzinotas, G. Zheng, and B. Ottersten, "Joint precoding with flexible power constraints in multibeam satellite systems," in IEEE Global Telecommunications Conference (GLOBECOM 2011), Houston, Texas, 2011.

[23] D. Christopoulos, S. Chatzinotas, and B. Ottersten, "Sum rate maximizing multigroup multicast beamforming under per-antenna power constraints," in Proc. of IEEE Global Communications Conference (GlobeCom), Austin, TX USA, Dec. 2014, Submitted. 